\title{
On the Surface: Reflectance FTIR Spectroscopy in Cultural Heritage Research
}

\author{
Joan Walker, Robyn Hodgkins and Barbara Berrie
}

National Gallery of Art, United States

FTIR spectroscopy is extremely useful for materials characterization in cultural heritage research, ${ }^{1}$ and various instrumental configurations are available (Table 1). A typical system found in a museum setting consists of a standard FTIR optical bench with $\mathrm{KBr}$ optics and liquid nitrogen-cooled mercury-cadmium-telluride (MCT) detector for fine spectral resolution. A FTIR microscope is frequently added for high spatial resolution. Portable spectroscopy systems are attractive for many applications: remote fieldwork, ${ }^{2}$ incorporation into scanning systems for mapping chemical constituents, ${ }^{3}$ and the in situ analysis of works of art in collection spaces. ${ }^{4,5}$ A truly portable spectrometer is modified to take advantage of the stability of Au mirror optics and ambient-temperature detectors, such as deuterated triglycine sulfate (DTGS). All instruments may be operated in various spectral acquisition modes. For cultural heritage purposes, more important distinctions amongst various modalities in FTIR spectroscopy are sampling vs. non-sampling and contact vs. non-contact. Reflectance spectroscopy is the least invasive method of collecting FTIR spectra. While a reflectance FTIR spectrum may be acquired on an excised sample (e.g. a mounted cross section), this modality allows for noncontact in situ analysis of works of art, a significant advantage in cultural heritage research.

Compared to transmission data, reflectance FTIR spectra can appear distorted. With judicious choices of reference materials and appropriate data manipulations, however, many of the challenges to using reflectance FTIR spectroscopy for material identification can be sufficiently addressed. For example, the Kramers-Kronig transformation (KKT) calculates a pseudo-absorbance spectrum that corrects some distortions. A successfully transformed spectrum can be directly compared to transmission data (Figure 1a) and is amenable to searches using spectral databases. ${ }^{6}$ Marker bands have been identified for many known materials ${ }^{7,8}$ to aid in determining their presence in distorted reflectance spectra without necessarily applying the KKT (Figure 1b). Multivariate methods such as principle component analysis may be useful to group materials that have similar spectra, given an appropriately chosen and sufficiently large set of references. ${ }^{9-11}$

Some factors to be aware of when translating between FTIR datasets collected using different modes are illustrated using spectra acquired from a small, well-characterized set of unvarnished contemporary oil paints of various pigment compositions and several examples of coated photographs. For paint samples, the $\mathrm{C}-\mathrm{H}$ stretching region is expected to be uncomplicated, consisting primarily of sharp symmetric and antisymmetric $\mathrm{C}-\mathrm{H}$ stretches in the hydrocarbon chains in linseed oil. However, in comparing the reflectance and transmission spectra of the entire set of samples, slight differences in band position and shape arise that appear to be unrelated to the sample composition (Figure 1c). The $\mathrm{C}=\mathrm{O}$ stretching region is more problematic. The strongly absorbing carbonyl groups generate derivative-like signals in reflectance, necessitating the use of the KKT for some spectral interpretations. Consequently, quantitative or semi-quantitative work using these vibrations is not straightforward. For example, the relationship between the oil ester $\left(1735 \mathrm{~cm}^{-1}\right)$ and free fatty acid $(1700$ $\mathrm{cm}^{-1}$ ) band intensities is not the same in the pseudo-absorbance spectra generated from reflectance data compared to the transmission spectra for the same samples (Figure 1d). Whether this is due to variations in surface vs. bulk concentrations of the relevant species or some other difference in the samples would need to be verified using additional analysis.

For stratified samples, surface-specific FTIR techniques such as attenuated total reflection (ATR) and external reflectance can provide depth-correlated spectral information. However, the presence of strongly absorbing layers may obscure underlying materials; and in external reflectance, the total signal (specular and diffuse) will be further affected by the surface roughness. ${ }^{12}$ Figure 2 shows how a thin surface coating may dominate the reflectance FTIR spectrum, in this case beeswax on different types of photographs. In all cases, the sharp 
bands due to beeswax are readily discernible in spectra collected in both ATR and reflectance modes on the coated samples. However, the ATR-FTIR spectra consistently show signals arising from cellulose and/or protein located underneath the applied coating. In the multi-layered silver gelatin developed out print, the baryta layer beneath the gelatin silver image layer is also detectable (Figure 2e). In reflectance mode, the sharp beeswax bands are enhanced, particularly in Figure 2f, where the specular reflectance of this glossy photograph dominates the spectrum.

FTIR spectroscopy is an important tool in material identification in cultural heritage research, though spectra collected using different modes, such as transmission, ATR, or reflectance, do not provide directly comparable results. An awareness of the spectral information accessed by different modes is imperative to making deductions about what chemical species are present, their concentrations, layering, and interactions. In reflectance, apparent shifts in peak positions and band widths can occur compared to transmission spectra; and reflectance FTIR will preferentially enhance signals from surface localized species depending on surface roughness, potentially obscuring materials in lower layers. The systematic study of the influence of highly absorbing constituents in complex mixtures and multi-layered structures will add to our ability to use these techniques to the best advantage to study cultural heritage. 
Table 1. Selection of FTIR instrument configurations used in cultural heritage research.

\begin{tabular}{|l|l|l|l|l|}
\hline Instrument Type & Sampling Modality & Accessories & Detector & Range $/ \mathbf{~ c m}^{-1}$ \\
\hline Benchtop & ATR & single-pass diamond/ZnSe crystal & DTGS & $4000-650$ \\
\hline Benchtop microscope & ATR & single-pass Ge crystal & MCT & $4000-800$ \\
\hline Benchtop microscope & Transmission & diamond cell & MCT & $4000-650$ \\
\hline Benchtop microscope & Reflectance & & MCT & $4000-600$ \\
\hline Stand-alone microscope & Reflectance & & MCT & $4000-750$ \\
\hline Benchtop fiber optic & Reflectance & chalcogenide fiber optic probe & MCT & $4000-900$ \\
\hline Compact portable & Reflectance & & DTGS & $7500-350$ \\
\hline
\end{tabular}

(a) FTIR Spectra of Oil Paint (Arylide Yellow)

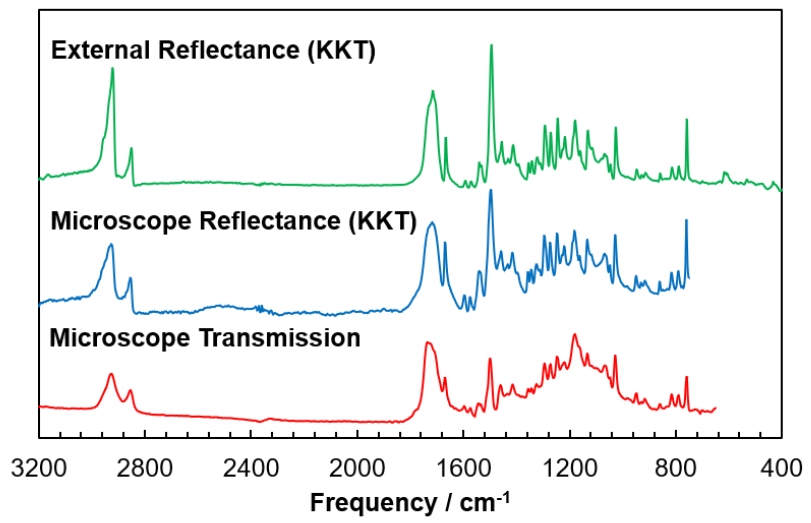

(b) FTIR Spectra of Oil Paint (Mars Black)

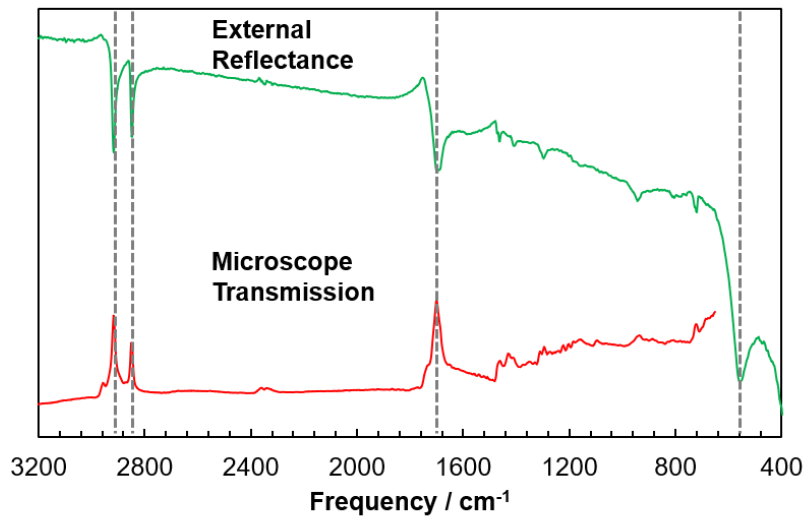

(c) Microscope

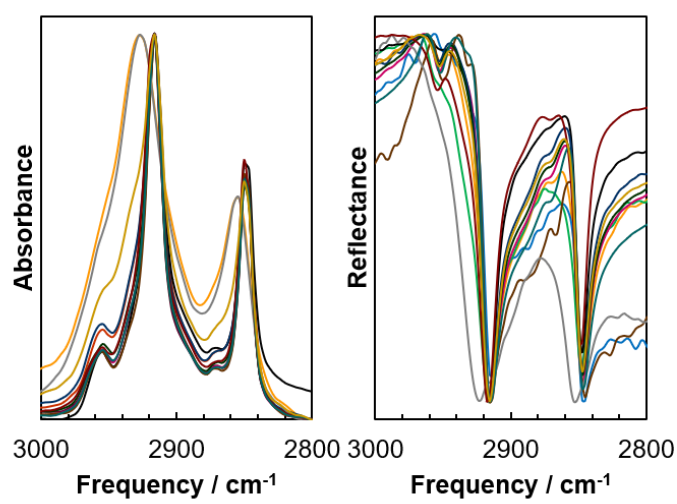

(d) Microscope

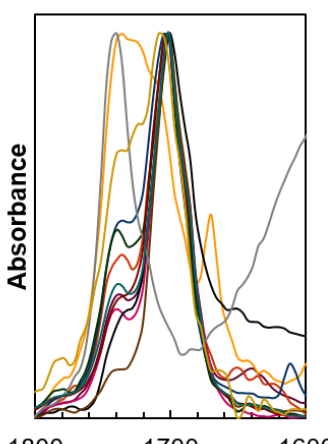

1800

Frequency $/ \mathrm{cm}^{-1}$

External Reflectance (KKT)

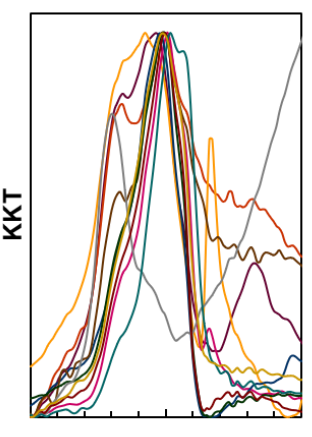

6001800

Frequency $/ \mathrm{cm}^{-1}$

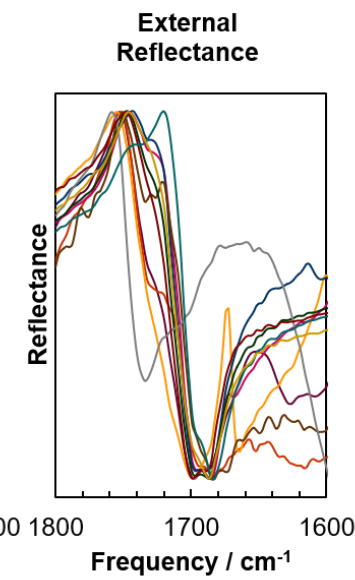

Figure 1. FTIR spectra of contemporary oil paints comparing transmission and reflectance modes. (a) The reflectance FTIR spectra of an arylide yellow paint are directly comparable to the transmission data using the KKT. (b) Peak positions for linseed oil and iron oxide are indicated and allow these components to be identified in both reflectance and transmission, regardless of the reflectance distortions. (c) The $\mathrm{C}-\mathrm{H}$ stretching region for the entire sample set shows that there is not a 1:1 correspondence between transmission and reflectance data, even in uncomplicated samples. (d) Distortions and changes in relative absorbances and peak widths between transmitted, reflected, and KK transformed infrared spectra are observed in the spectroscopically rich $\mathrm{C}=\mathrm{O}$ stretching region. 
(a) Salted Paper Prints: ATR FTIR Spectra

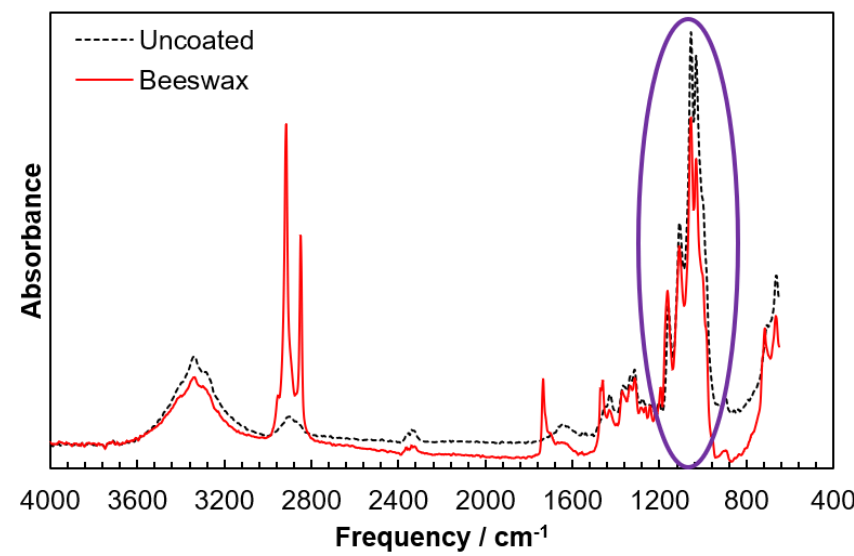

(c) Gelatin-sized Prints: ATR FTIR Spectra

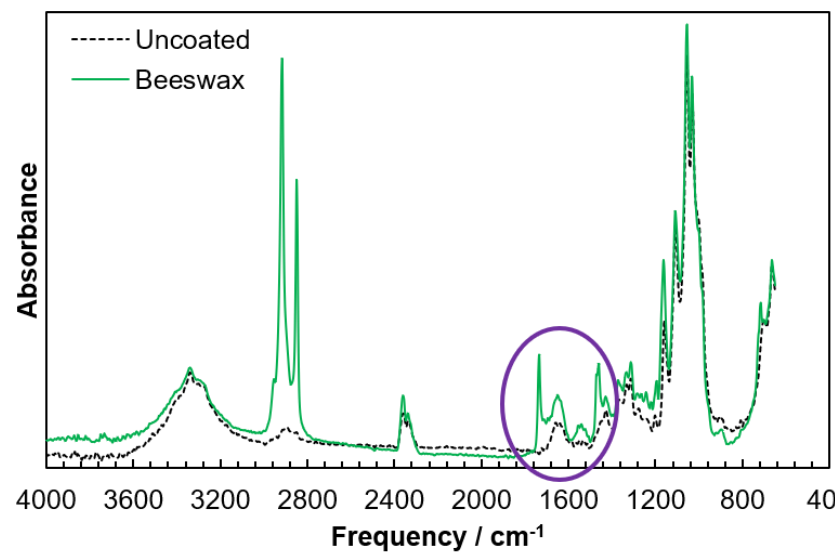

(e) Silver Gelatin Prints: ATR FTIR Spectra

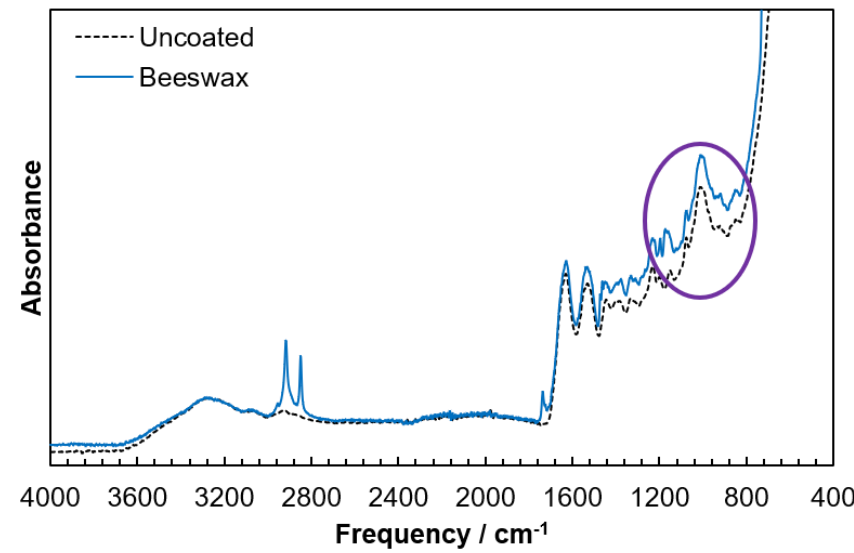

(b) Salted Paper Prints: Reflectance FTIR Spectra

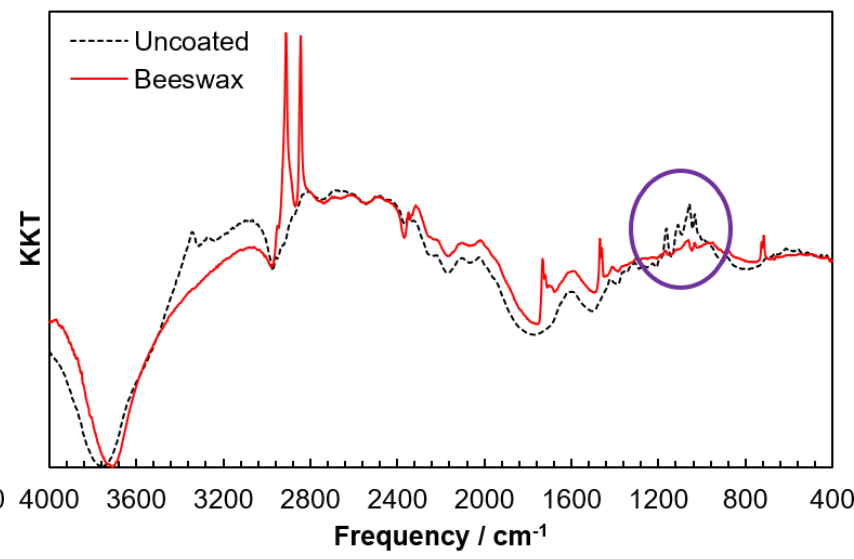

(d) Gelatin-sized Prints: Reflectance FTIR Spectra

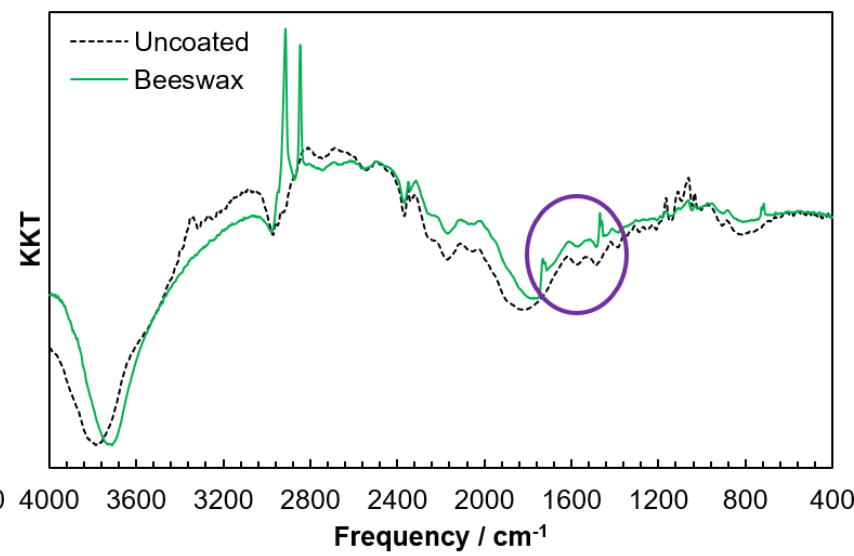

(f) Silver Gelatin Prints: Reflectance FTIR Spectra

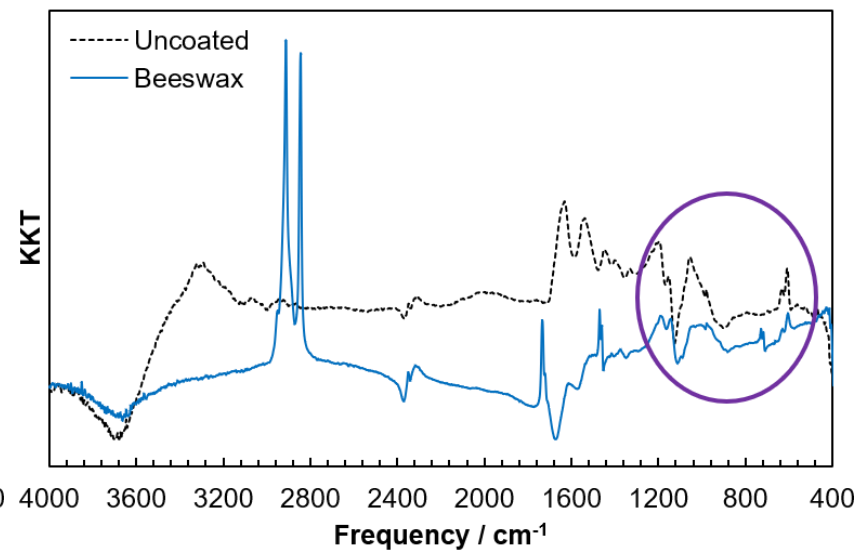

Figure 2. Comparisons of FTIR spectra in ATR and reflectance modes of uncoated and beeswax-coated photographs. (a, b) Salted paper prints on paper (cellulose peaks circled). (c, d) Pd prints on gelatin-sized paper (protein bands circled). (e, f) Silver gelatin developed out prints with baryta underlayer (barium sulfate region circled).

\section{References}


1. Derrick, M. R.; Stulik, D.; Landry, J. M., Infrared Spectroscopy in Conservation Science. Getty Conservation Institute: Los Angeles, 1999.

2. Miliani, C.; Rosi, F.; Borgia, I.; Benedetti, P.; Brunetti, B. G.; Sgamellotti, A. Applied Spectroscopy 2007,61 (3), 293-299.

3. Legrand, S.; Alfeld, M.; Vanmeert, F.; De Nolf, W.; Janssens, K. Analyst 2014,139 (10), 2489-2498.

4. Bell, J.; Nel, P.; Stuart, B. Heritage Science 2019,7 (1), 95.

5. Saviello, D.; Toniolo, L.; Goidanich, S.; Casadio, F. Microchemical Journal 2016,124, 868-877.

6. Picollo, M.; Bartolozzi, G.; Cucci, C.; Galeotti, M.; Marchiafava, V.; Pizzo, B. Applied Spectroscopy 2014,68 (4), 389-397.

7. $\quad$ Miliani, C.; Rosi, F.; Daveri, A.; Brunetti, B. G. Applied Physics A 2012,106 (2), 295-307.

8. Rosi, F.; Daveri, A.; Moretti, P.; Brunetti, B. G.; Miliani, C. Microchemical Journal 2016,124, 898908.

9. $\quad$ Carter, E. A.; Swarbrick, B.; Harrison, T. M.; Ronai, L. Heritage Science 2020,8 (1), 51.

10. Bacci, M.; Fabbri, M.; Picollo, M.; Porcinai, S. Analytica Chimica Acta 2001,446 (1), 15-21.

11. McClelland, A.; Bulat, E.; Bernier, B.; Murphy, E. L. Journal of the American Institute for Conservation 2020,59 (2), 123-136.

12. Vetter, W.; Latini, I.; Schreiner, M. Heritage Science 2019,7 (1), 21. 